\title{
Correction to: Association of Allergic Symptoms with Dengue Infection and Severity: A Systematic Review and Meta-analysis
}

\author{
Nguyen Dang Kien ${ }^{1,2} \cdot$ Amr Ehab El-Qushayri ${ }^{2,3} \cdot$ Ali Mahmoud Ahmed ${ }^{2,4} \cdot$ Adnan Safi $^{2,5} \cdot$ Sarah Abdel Mageed ${ }^{2,6}$. \\ Samar Muhammed Mehyar ${ }^{2,7} \cdot$ Mohammad Rashidul Hashan $^{2,8} \cdot$ Sedighe Karimzadeh $^{2,9} \cdot$ Kenji Hirayama $^{10}$. \\ Nguyen Tien Huy ${ }^{11,12}$
}

Published online: 21 December 2019

(C) Wuhan Institute of Virology, CAS 2019

\section{Correction to: Virologica Sinica}

https://doi.org/10.1007/s12250-019-00165-6

The spelling of the fifth author's name was misspelled. The byline should appear as shown above. The original article has been corrected.

The original article can be found online at https:// doi.org/10.1007/s12250-019-00165-6.

Nguyen Tien Huy

nguyentienhuy@tdtu.edu.vn

1 Department of Obstetrics and Gynecology, Thai Binh University of Medicine and Pharmacy, Thai Binh 410000, Vietnam

2 Online Research Club (http://www.onlineresearchclub.org/), Nagasaki, Japan

3 Faculty of Medicine, Minia University, Minia 61519, Egypt

4 Faculty of Medicine, Al-Azhar University, Cairo 11865 , Egypt

5 Nishtar Medical University, Multan 60000, Pakistan

6 Faculty of Medicine, Tanta University, Tanta 31511, Egypt
Istishari Hospital, Amman 11953, Jordan

8 Infectious Disease Division, Respiratory and Enteric Infections Department, International Center for Diarrheal Disease Research, Dhaka, GPO Box 128, Dhaka 1000, Bangladesh

9 School of Medicine, Sabzevar University of Medical Sciences, Sabzevar 00989156113915, Iran

10 Department of Immunogenetics, Institute of Tropical Medicine (NEKKEN), Leading Graduate School Program, and Graduate School of Biomedical Sciences, Nagasaki University, 1-12-4 Sakamoto, Nagasaki 852-8523, Japan

11 Evidence Based Medicine Research Group, Ton Duc Thang University, Ho Chi Minh City 70000, Vietnam

12 Faculty of Applied Sciences, Ton Duc Thang University, Ho Chi Minh City 70000, Vietnam 